\title{
The Northwestern Abdominoplasty Model - A novel human scar model
}

\author{
Ji-Cheng Hsieh, BA, Chitang J. Joshi, MBBS MS, Rou Wan, MD, Robert D. Galiano, MD, FACS* \\ Department of Plastic and Reconstructive Surgery, Feinberg School of Medicine, Northwestern University, Chicago, IL
}

${ }^{*}$ Corresponding author: Dr. Robert Galiano, MD., FACS, 675 N. St. Clair St. Galter 19-250, Chicago, IL, 60611 , Tel: 312-695-1736; E-mail: r-galiano@northwestern.edu

Received: May 15, 2020; Accepted: May 21, 2020; Published: May 25, 2020

\section{Editorial}

Scarring is a cosmetically undesirable outcome of wound healing. Modern scar research investigates the processes behind scarring and, more importantly, the efficacy of medications and procedures. The wide variety of scar models ranges from animal models - red duroc pigs, athymic mice, rabbit ears - to virtual "in silico" models, to human cell models of varying complexity in regards to mimicking the true in vivo environment. Current volunteer or patient human models of scarring are limited by ethical concerns regarding purposeful wounding, difficulty controlling factors such as location, scar size, and scar orientation in existing scars, and, in general, the challenges of recruitment for a human scar study.

The Northwestern Abdominoplasty Scar Model was introduced in 2016 and touted to be a streamlined and effective modern in vivo human scar model [1]. To better characterize the role of the model in current scar research, Hsieh and colleagues published a summary article comparing the Northwestern Abdominoplasty Scar Model to the existing gamut of scar models, especially new developments in animal models [2]

The Northwestern Abdominoplasty Scar Model comprises of patients undergoing elective abdominoplasty. After a detailed and thorough informed consent process, patients who agree are scarred or wounded in the pannus using local anesthesia in the clinic. As the pannus is typically large, 202 -cm full-thickness horizontal wounds are able to be produced in a grid fashion. The wounds are created to mirror each other in location and, as a result, one side can test the trial intervention while the other serves as the control. Outcome measures include visual assessment of scarring with pictures and histological and biochemical analysis through biopsy. Weeks to months after the end of the study, the pannus removed as a part of the abdominoplasty.

The main benefit of the Northwestern Abdominoplasty scar model, compared to animal, in silico, or in vitro models is that the model utilizes scarring in humans. Compared to other volunteer or patient in vivo models, the Northwestern Abdominoplasty Scar Model overcomes the primary limitations of 1) control in wound characteristics, 2) patient recruitment and 3) ethical considerations surrounding purposeful wounding.
Factors such as the size, number, and types of wounds created can be altered according to the investigator's specifications. Multiple interventions at various doses can be tested using the same patient. Since the abdominoplasty is a fairly common cosmetic procedure and that the procedure is cost-free should a patient choose to participate, recruitment is streamlined, and since each patient can be wounded multiple times, studies can require fewer patients. Ethical hurdles of purposeful scarring and potentially leaving patients with wounds or scars are bypassed given that the pannus is excised during the abdominoplasty.

The primary limitation of the model rests in performing a detailed informed consent process and emphasizing to the patient that should they opt out of the study, the scars created will remain. Another major limitation is that only low-tension, ventral abdominal wounds can be studied. It is difficult to assess pathological scarring in this model, as patients who present for elective abdominoplasty may or may not scar well, and patients with history of keloids are specifically excluded out of concern for poor scarring. Other limitations include efficacy in assessing deep, vertical, or curved wounds, as only shallow, linear scars have been studied using this model. Further studies of this model will characterize its role in studying these wounds, especially given that tension varies depending on the orientation. Overall, the Northwestern Abdominoplasty Model offers several critical improvements over existing in vivo human models, but will encourage greater interest in in vivo human model development, with potential utilization of cosmetic surgeries in other areas of the body for scar research.

Keywords: Plastic surgery, scars, scar models, wound, wound healing, review

\section{References}

1. Lanier ST, Liu J, Chavez-Munoz C, Mustoe TA, Galiano RD (2016) The Northwestern Abdominoplasty Scar Model: A Novel Human Model for Scar Research and Therapeutics. Plast Reconstr Surg Glob Open. 4(9):e867. [crossref]

2. Ji-Cheng Hsieh, BA; Chitang J. Joshi, MBBS MS; Rou Wan, MD; Robert D. Galiano, MD, FACS (2020) The Northwestern Abdominoplasty Scar Model: A Tool for Highthroughput Assessment of Scar Therapeutics. Advances in Wound Care. 00(00):0-0 [crossref]

Citation:

Ji-Cheng Hsieh, Chitang J. Joshi, Rou Wan, Robert D. Galiano (2020) The Northwestern Abdominoplasty Model - A novel human scar model.. J Clin Res Med Volume 3(2): 1-1. 